\title{
Acute Limb Ischemia in a Previously Undiagnosed Case of Mid-Ventricular Non-Obstructive Hypertrophic Cardiomyopathy
}

\author{
Imo Ebonga, b, d, John Rozich ${ }^{\mathrm{a}, \mathrm{b}}$, Madhan Shanmugasundaram ${ }^{\mathrm{a}, \mathrm{b}}$, Zachary Taylor ${ }^{\mathrm{a}, \mathrm{b}, \mathrm{c}}$, \\ Rajesh Janardhanan ${ }^{\mathrm{a}, \mathrm{b}}$, Divya Kapoor ${ }^{\mathrm{a}, \mathrm{b}}$
}

\begin{abstract}
Mid-ventricular hypertrophic cardiomyopathy (HCM) is rare and when associated with an apical aneurysm has a high risk of thrombus formation and embolization. We report a case of a 70-year-old man with peripheral vascular disease who presented with acute progressive right leg pain. Peripheral angiography showed complete thrombotic occlusion of his right superficial femoral artery. Thrombolysis was successfully performed using alteplase. His post-procedure angiography which was obtained the next day showed complete revascularization with no residual stenosis. His echocardiogram showed an ill-defined mass in the left ventricular apex. The absence of residual atheroma on peripheral angiography suggested a primary embolic event. We obtained cardiac MRI which showed mid-ventricular HCM complicated by an apical aneurysm and small thrombus. Left ventricular apical aneurysm occurs commonly in mid-ventricular HCM and is associated with a worse prognosis. Cardiac magnetic resonance imaging (MRI) is superior to echocardiography in identifying left ventricular apical aneurysms and apical thrombi.
\end{abstract}

Keywords: Hypertrophic cardiomyopathy; Thromboembolism; Apical aneurysm

\section{Introduction}

Hypertrophic cardiomyopathy $(\mathrm{HCM})$ is a genetic disorder with segmental myocardial hypertrophy, varying disease expression and complications [1]. Most patients are diagnosed

Manuscript submitted September 11, 2017, accepted September 18, 2017

aDepartment of Cardiology, University of Arizona College of Medicine/Sarver Heart Center, Tucson, AZ, USA

bDepartment of Cardiology, Southern Arizona Veteran Affairs Health Care System, Tucson, AZ, USA

'Department of Vascular Surgery, Southern Arizona Veteran Affairs HealthCare System, Tucson, AZ, USA

${ }^{\mathrm{d} C}$ Corresponding Author: Imo Ebong, Division of Cardiology, University of Arizona College of Medicine, 1501 North Campbell Avenue, Tucson, AZ, USA. Email: iebong@shc.arizona.edu

doi: https://doi.org/10.14740/jmc2915w with asymmetric septal hypertrophy and less commonly with the mid-ventricular, apical, posterior-septal or isolated lateral wall types [2]. The mid-ventricular variant can either present as an obstructive or non-obstructive disease, with complications such as left ventricular (LV) apical aneurysm, thrombosis and embolization [1,3]. The mechanisms of aneurysm formation are incompletely understood [4], but its presence is associated with higher risks of complications $[4,5]$. We report a case of mid-ventricular HCM with LV apical aneurysm presenting with acute limb ischemia of right lower extremity. Another interesting aspect of this case was that the LV apical aneurysm, associated residual thrombus and mid-ventricular HCM variant were not apparent on echocardiography but were diagnosed by cardiac magnetic resonance imaging (MRI).

\section{Case Report}

A 72-year-old African-American man was admitted with complaints of acute progressive right leg pain of 4 days duration. He had a past medical history of hypertension, hyperlipidemia, chronic kidney disease and peripheral vascular disease with prior stent placement in his left superficial femoral artery (SFA). He had a normal right femoral pulse but no palpable distal pulses. We were unable to document popliteal, posterior tibial or dorsalis pedis pulse by hand held Doppler. He was started on systemic anticoagulation and referred for an emergent peripheral angiogram. Peripheral angiography showed an angiographically normal appearing right common femoral artery and profunda branch. The SFA was occluded in the proximal segment with no visualization of the distal vessels (Fig. 1a). Catheter-directed thrombolytic therapy was successfully completed with alteplase. The post-procedure angiogram obtained the following day showed complete recanalization of his SFA with no residual thrombus or atheroma (Fig. 1b). Telemetry monitoring and electrocardiography showed no evidence of arrhythmia. Echocardiography showed a preserved LV ejection fraction of 55-60\% with normal segmental wall motion and mild LV hypertrophy (LVH). There was an ill-defined mass in the LV apex (Fig. 2a). Although the initial diagnosis suggested atherothrombotic disease, the absence of residual disease after thrombolysis increased our suspicion for a thrombotic event. A computed tomography scan was negative for aortic thrombus but an incidental finding was made of focal 


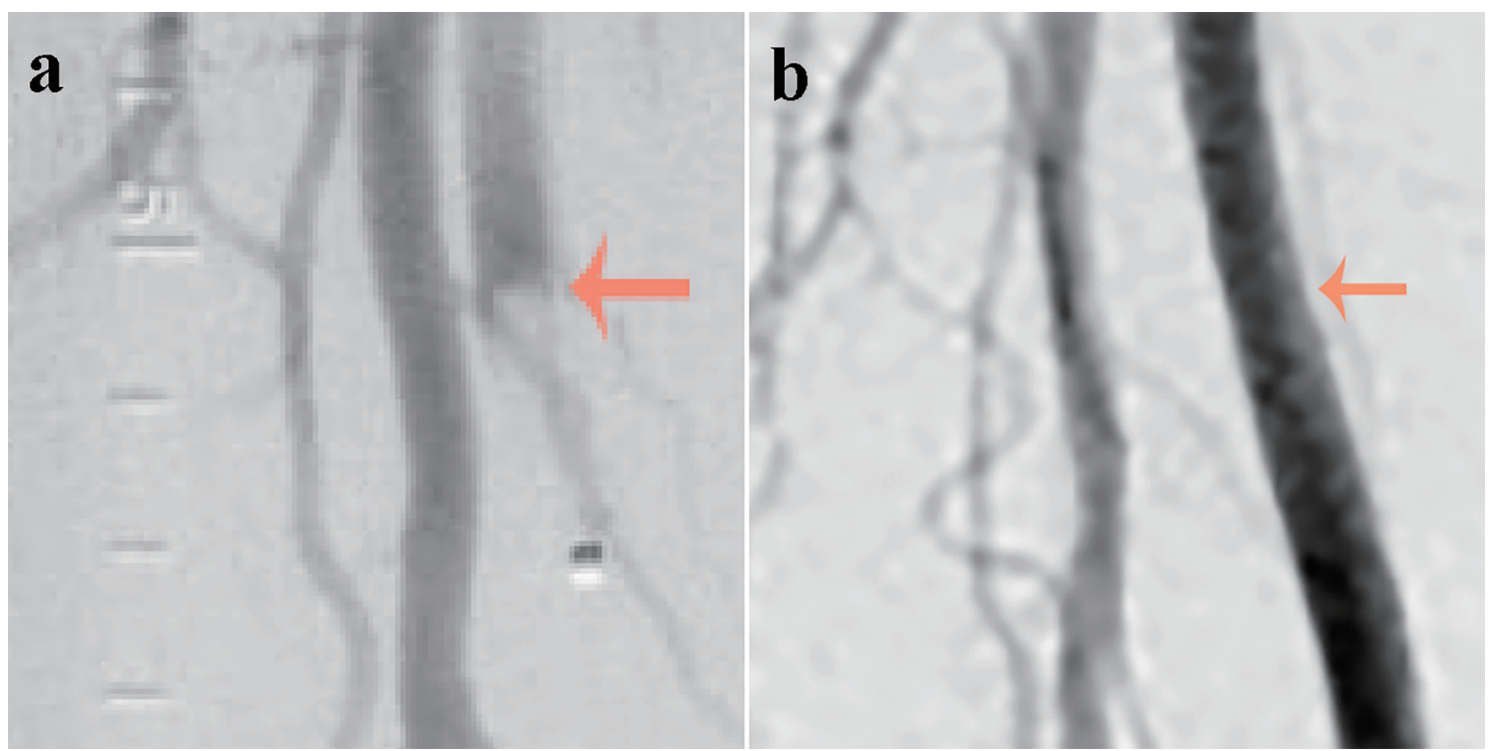

Figure 1. (a) An abrupt occlusion (indicated by an arrow) in the superficial femoral artery just distal to its bifurcation with a positive meniscus sign (image on the left obtained at presentation). (b) Complete recanalization of the superficial femoral artery with no residual thrombus or atheroma (image on the right obtained after thrombolysis).

thinning in the LV apex. A contrast-enhanced echocardiogram confirmed the presence of an apical density (Fig. 2b). This was followed by a cardiac MRI which confirmed the diagnosis of mid-ventricular HCM with LV apical aneurysm and a small residual thrombus (Fig. 3). He was discharged home on anticoagulation (warfarin) therapy and has had no further embolic episodes on follow-up.

\section{Discussion}

HCM is a clinical entity that is associated with unexplained LVH and non-dilated ventricular chambers in the absence of other cardiac causes that could result in a similar magnitude of hypertrophy [6]. Apical aneurysms can occur in approximately $25 \%$ of HCM patients [7]. Although aneurysms can occur in non-obstructive HCM, they are more common in those with mid-ventricular obstruction $[7,8]$. The mechanisms of apical aneurysm formation are incompletely understood but include increased intraventricular pressure, microvascular ischemia, left anterior descending artery bridging and genetic predisposition $[1$, 4, 7, 8]. Patients may be asymptomatic [7], but the presence of aneurysm is associated with increased risk of complications [1, $4,7]$ particularly when they are medium or large-sized [9]. The dyskinetic or akinetic apical aneurysm provides a structural basis for intracavitary thrombus formation [5]. The diagnostic accuracy for $\mathrm{LV}$ apical aneurysm is $57 \%$ for echocardiogram, $80 \%$ for contrast echocardiography and 100\% for cardiac MRI [9].

In older patients, the likelihood of HCM is increased by the presence of marked LV thickness $>25 \mathrm{~mm}$, LV outflow tract (LVOT) obstruction, systolic anterior motion of the mitral valve and/or the presence of mitral valve to septal wall contact [6]. None of these features were present in our patient who failed to meet the traditional diagnostic criteria of HCM by echocardiography. Elderly HCM patients like ours are more likely to have milder hypertrophy with coexisting morbidities like hy-

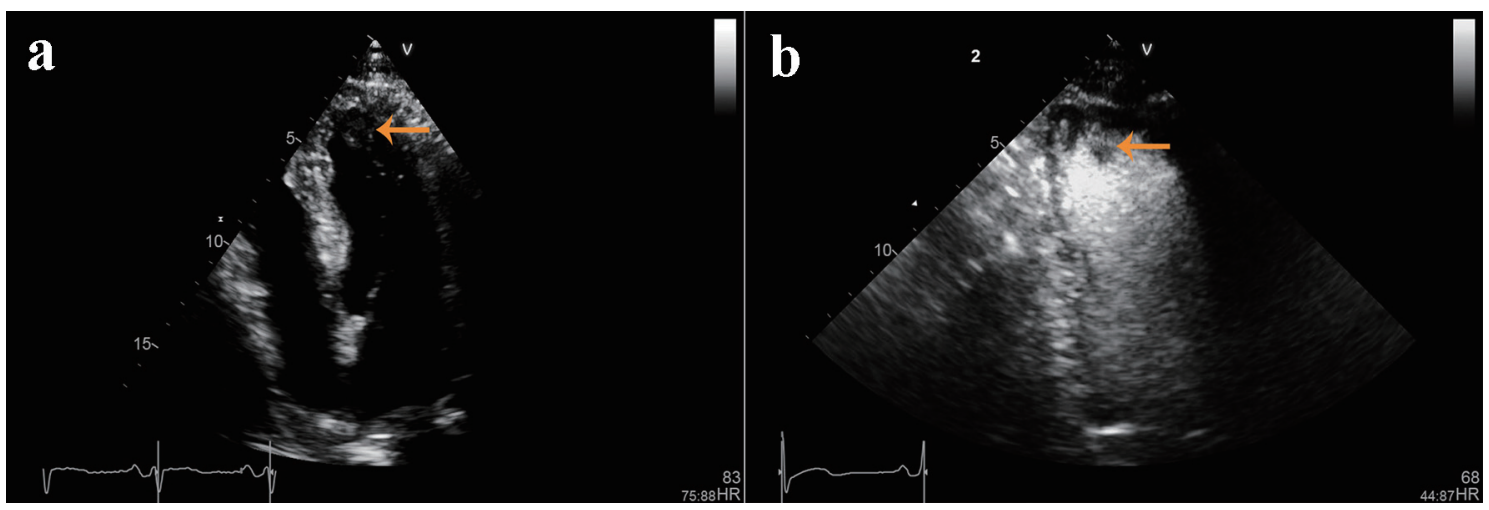

Figure 2. (a) Non-contrast and (b) contrast-enhanced echocardiographic images showing left ventricular hypertrophy and an ill-defined mass in the left ventricular apex. 


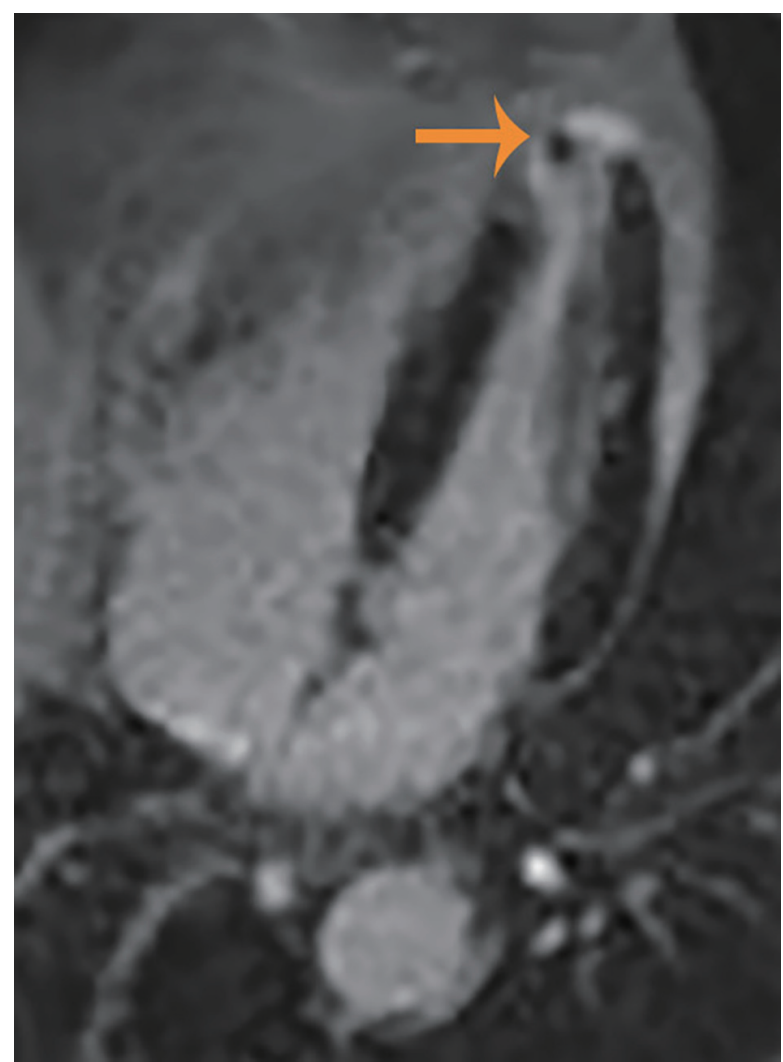

Figure 3. Cardiac magnetic resonance image showing left ventricular hypertrophy, small aneurysm and thrombus at the left ventricular apex.

pertension or aortic stenosis which by themselves could cause LV hypertrophy [7]. Our patient had LVH which was graded as mild and attributed to systemic hypertension. The basal septum dimension was measured at $14 \mathrm{~mm}$ with no intracavitary gradients or aneurysms on echocardiography. An ill-defined density was visualized on both the contrast and non-contrast echocardiographic images but no definite evidence of thrombus was seen.

It would be unusual to have a thrombus in the left ventricle with normal contractile function and no demonstrable wall motion abnormalities. Therefore, we obtained cardiac MRI to further delineate the apical density. Cardiac MRI is useful to demonstrate myocardial scarring by late gadolinium enhancement (LGE) especially when apical aneurysm is present [6]. LGE was not demonstrated in the LV apex or aneurysmal segment in our patient. The absence of LGE may indicate a variable pattern of myocardial involvement which could provide clues to the mechanisms of aneurysm formation [4]. Although not necessary for diagnosis, cardiac MRI may be an important part of a multimodality approach in evaluating patients with HCM $[2,4,10]$ particularly when complications are suspected. Cardiac MRI may be more sensitive in the diagnosis of elderly patients and those with mild variants of the disease.

Patients with mid-ventricular obstruction are usually recognized sooner because they have symptoms more often than those without obstruction or those with LVOT obstruction [8]. Symptoms are also more common in HCM patients with moderate degrees of hypertrophy (wall thickness of $16-24 \mathrm{~mm}$ ) as opposed to those with severe hypertrophy $(>30 \mathrm{~mm})$ or mild hypertrophy $(<15 \mathrm{~mm})$ [9] as was seen in our patient. Patients could experience syncope, palpitations, chest pain or dyspnea while others may present with resuscitated cardiac arrest even in the absence of a family history of sudden cardiac death. A coronary angiogram or myocardial perfusion imaging should be pursued in patients with HCM who have chest pain and an intermediate or high risk of coronary artery disease (CAD) [6].

Although assessment for silent ischemia is not indicated in asymptomatic patients [6], this should be considered in patients with cardiovascular disease risk factors when an apical aneurysm is present. Stress myocardial perfusion imaging in patients with HCM may show reversible or fixed perfusion defects consistent with ischemia or infarction even when epicardial CAD is absent [6]. Our patient had stress myocardial perfusion imaging performed as part of a preoperative evaluation and was incidentally reported to have a small infarct in the anterior septum with no reversible ischemia. However, the absence of myocardial scarring or LGE on cardiac MRI implied that this was unlikely to be a result of ischemic disease.

Although late-onset HCM has a better prognosis than HCM in younger individuals, it could still result in progressive disease [7] and lead to complications as illustrated in this case. Known high risk markers in HCM include personal or family history of sudden cardiac death events, history of unexplained syncope or malignant arrhythmias, documented non-sustained ventricular tachycardia on ambulatory electrocardiographic monitoring, abnormal blood pressure response recorded during exercise and a maximum LV wall thickness $\geq 30 \mathrm{~mm}$. The combined annual event rate of complications can be as high as $10 \%$ when apical aneurysms are present but apical aneurysms are not yet recognized as a high risk marker in HCM [7]. The patient presented in this case had a small apical aneurysm but failed to exhibit any other high risk markers for sudden cardiac death.

\section{Conclusion}

Non-obstructive mid-ventricular HCM is a rare disease that is associated with a high risk of complications particularly when an LV apical aneurysm is present. Aneurysms may cause thrombus formation which may result in thromboembolic complications. Cardiac MRI is useful in elderly patients with mild unexplained hypertrophy when echocardiography is inconclusive or when complications such as aneurysm or thrombus are suspected.

\section{Prior Presentation}

This paper was presented as a poster at the Annual Scientific Sessions of the American College of Cardiology 2017 Conference in Washington, DC.

\section{Funding}

This research did not receive any specific grant from fund- 
ing agencies in the public, commercial, or not-for-profit sectors

\section{Conflict of Interest}

The authors declare that there is no conflict of interest regarding the publication of this article.

\section{References}

1. Xiao Y, Wang LP, Yang YK, Tian T, Yang KQ, Sun X, Jiang Y, et al. Clinical profile and prognosis of left ventricular apical aneurysm in hypertrophic cardiomyopathy. Am J Med Sci. 2016;351(1):101-110.

2. Sivanandam A, Ananthasubramaniam K. Midventricular hypertrophic cardiomyopathy with apical aneurysm: potential for underdiagnosis and value of multimodality imaging. Case Rep Cardiol. 2016;2016:9717948.

3. Saba SG, Ertel AW, Siegenthaler M, Bodurian E, Kellman P, Chen MY, Arai AE, et al. Hemodynamic consequences of hypertrophic cardiomyopathy with midventricular obstruction: apical aneurysm and thrombus formation. J Gen Pract (Los Angel). 2014;2(4):161.

4. Rajani R, De Silva K, Brum R, Hancock J. Multimodality imaging of the ace of spades. J Cardiovasc Med (Hagerstown). 2010;11(2):137-139.

5. Maron MS, Finley JJ, Bos JM, Hauser TH, Manning WJ, Haas TS, Lesser JR, et al. Prevalence, clinical significance, and natural history of left ventricular apical aneurysms in hypertrophic cardiomyopathy. Circulation.
2008;118(15):1541-1549.

6. Gersh BJ, Maron BJ, Bonow RO, Dearani JA, Fifer MA, Link MS, Naidu SS, et al. 2011 ACCF/AHA Guideline for the Diagnosis and Treatment of Hypertrophic Cardiomyopathy: a report of the American College of Cardiology Foundation/American Heart Association Task Force on Practice Guidelines. Developed in collaboration with the American Association for Thoracic Surgery, American Society of Echocardiography, American Society of Nuclear Cardiology, Heart Failure Society of America, Heart Rhythm Society, Society for Cardiovascular Angiography and Interventions, and Society of Thoracic Surgeons. J Am Coll Cardiol. 2011;58(25):e212-260.

7. Bonou M, Papadimitraki ED, Dalianis N, Alexopoulos N, Karali M, Zerva K, Barbetseas J. Apical aneurysm with thrombus in an elderly patient with hypertrophic obstructive cardiomyopathy. Aging Clin Exp Res. 2017;29(2):341-344.

8. Efthimiadis GK, Pagourelias ED, Parcharidou D, Gossios T, Kamperidis V, Theofilogiannakos EK, Pappa Z, et al. Clinical characteristics and natural history of hypertrophic cardiomyopathy with midventricular obstruction. Circ J. 2013;77(9):2366-2374.

9. Parato VM, Antoncecchi V, Sozzi F, Marazia S, Zito A, Maiello M, Palmiero P, et al. Echocardiographic diagnosis of the different phenotypes of hypertrophic cardiomyopathy. Cardiovasc Ultrasound. 2016;14(1):30.

10. Rowin EJ, Maron BJ, Haas TS, Garberich RF, Wang W, Link MS, Maron MS. Hypertrophic cardiomyopathy with left ventricular apical aneurysm: implications for risk stratification and management. J Am Coll Cardiol. 2017;69(7):761-773. 